УДК 517.98

\author{
O. V. LOPOTKO
}

\title{
EVENLY POSITIVE DEFINITE FUNCTION OF HILBERT SPACE AND SOME ALGEBRAIC RELATIONSHIP
}

\begin{abstract}
O. V. Lopotko. Evenly positive definite function of Hilbert space and some algebraic relationship, Mat. Stud. 55 (2021), 85-93.

A generalization of P. A. Minlos, V. V. Sazonov's theorem is proved in the case of bounded evenly positive definite function given in a Hilbert space. The integral representation is obtained for a family of bounded commutative self-adjoint operators which are connected by algebraic relationship.
\end{abstract}

This scientific work is devoted to 95-th anniversary of Professor Yu. M. Berezansky

For the first time theorem about integral representation for positive definite (p.d.) functions $k(x)\left(x \in \mathbb{R}^{1}\right)$ was obtained in papers of Krein M. G. and Berezansky Yu. M. $[1,5]$. Then Berezansky Yu. M. obtained the integral representation for functions $k(x)\left(x \in \mathbb{R}^{n}\right)$ [2]. In the next investigations Berezansky Yu. M. used methods of the spectral theory of operators. These methods play an important role in infinite-dimensional analysis. Using these methods Berezansky Yu. M. obtained the integral representation for p.d. functions in the space $(-2 l ; 2 l) \times \mathbb{R}^{1} \times \mathbb{R}^{1} \times \ldots$ and in the Hilbert space $H_{2 l}$ [4]. In particular, for every p.d. continuous in $j$-topology function $k(x)\left(x \in H_{2 l} ; l \leq \infty\right)$ the following integral representation is valid

$$
k(x)=\int_{H} e^{i(\lambda, x)} d \rho(\lambda), \quad\left(x \in H_{2 l}\right) .
$$

Here $d \rho(\lambda)$ is a non-negative finite measure on some $\sigma$-algebra of Borel sets from $H$. Conversely, every integral of form (1) is a p.d. function in $H$ which is continuous at $O$ in $j$-topology. In the case of $l=\infty$, the measure $d \rho(\lambda)$ is uniquely determined by $k(x)$; in the case of $l<\infty$, there is no uniqueness.

The article presents a modification of this theory to the case of bounded evenly p.d. functions $k(x)(x \in H)$.

Let $H$ be a real valued separable Hilbert space. A real convex bounded even function $k(x)(x \in H)$ is called positive definite (e.p.d.), if for $x^{(1)}, \ldots, x^{(N)} \in H$ and $\xi_{1}, \ldots, \xi_{N} \in \mathbb{C}^{1}$ $(n=N)$ the inequality

$$
\sum_{j, \kappa=1}^{N} \frac{1}{2}\left[k\left(x^{(j)}+x^{(\kappa)}\right)+k\left(x^{(j)}-x^{(\kappa)}\right)\right] \xi_{j} \overline{\xi_{\kappa}} \geq 0
$$

2010 Mathematics Subject Classification:46E20, 47G10.

Keywords: integral representation; bounded evenly positive definite functions; bounded self-adjoint operators. doi:10.30970/ms.55.1.85-93

(C) O. V. Lopotko, 2021 
holds.

Let $\mathcal{A}$ be a nonnegative nuclear operator. A topology given by neighborhoods of the origin of the form $\left\{x \in H \mid(\mathcal{A} x, x)_{H}<\varepsilon\right\}(\varepsilon>0)$ is called the $j$-topology in $H$. A function $k(x)(x \in H)$ is said to be continuous at the origin in the $j$-topology if it is continuous at $O$ in the topology inducted by the $j$-topology on $H$.

Theorem 1. Let $k(x)(x \in H)$ be any bounded e.p.d. function which is continuous at $O$ in the $j$-topology. Then the function admits the representation

$$
k(x)=\int_{H} \cos (\lambda, x) d \rho(\lambda) \quad(x \in H),
$$

where $d \rho(\lambda)$ is a non-negative finite measure defined on the $\sigma$-algebra $\mathfrak{B}(H)$ of Borel subsets of $H$. Conversely, each integral of the form (3) is a bounded e.p.d. function in $H$ which is continuous at the origin in the $j$-topology. The measure $d \rho(\lambda)$ is uniquely defined for a given $k$.

We will prove that $(2)$ implies the following inequality:

1) If $\sup |k(x)|=C<+\infty$ then $|k(x)| \leq|k(0)|$, i.e. $C=|k(0)|$.

Indeed, put in $(2) N=2, \xi_{1}=-1, \xi_{2}=1, x^{(1)}=x, x^{(2)}=0$. Then $3 k(0)+k(2 x)-$ $4 k(x) \geq 0$. It follows that $k(2 x)+3 k(0) \geq 4 k(x)$ and

$$
|k(x)| \leq \frac{|k(2 x)|+3|k(0)|}{4} \leq \frac{3|k(0)|+C}{4}, \quad C=\sup |k(x)| \leq \frac{3|k(0)|+C}{4}
$$

It means that $C \leq|k(0)|$, and therefore

$$
|k(x)| \leq|k(0)|
$$

2) For an arbitrary convex bounded e.p.d. function the following inequality holds

$$
\left|k\left(x^{(1)}\right)-k\left(x^{(2)}\right)\right|^{2} \leq 2 k(0)\left\{k(0)-k\left(x^{(1)}-x^{(2)}\right)\right\} .
$$

Note that for an arbitrary p.d. kernel $K(x ; y)$ we have (see [4, p. 469, Lemma 4.1])

$$
|K(x ; z)-K(y ; z)|^{2} \leq K(z ; z)[K(x ; x)-2 \operatorname{Re} K(x ; y)+K(y ; y)]
$$

Put in this inequality $K(x ; y)=\frac{1}{2}\left[k\left(x^{(1)}-x^{(2)}\right)+k\left(x^{(1)}+x^{(2)}\right)\right](z=0)$. We obtain

$$
\begin{aligned}
& \left|k\left(x^{(1)}\right)-k\left(x^{(2)}\right)\right|^{2} \leq k(0)\left[k(0)+\frac{1}{2} k\left(2 x^{(1)}\right)+\right. \\
& \left.+\frac{1}{2} k\left(2 x^{(1)}\right)-k\left(x^{(1)}-x^{(2)}\right)-k\left(x^{(1)}+x^{(2)}\right)\right] .
\end{aligned}
$$


But

$$
\frac{1}{2}\left(k\left(2 x^{(1)}\right)+k\left(2 x^{(2)}\right)\right)-k\left(x^{(1)}+x^{(2)}\right) \leq 0,
$$

because the function $k(x)$ is convex. Therefore the previous inequality can be rewritten in the form

$$
\left|k\left(x^{(1)}\right)-k\left(x^{(2)}\right)\right|^{2} \leq k(0)\left\{k(0)-k\left(x^{(1)}-x^{(2)}\right)\right\} .
$$

Proof of Theorem 1. Using (4), (5) (see [4, p.467-469]) we can prove that there exists a nonnegative nuclear operator $\mathcal{A}$ in $H$ with kernel of zero such that $k(x)(x \in H)$ is uniformly continuous in the following sense: for any $\varepsilon>0$ there exists $\delta>0$ such that for $x^{(1)}, x^{(2)} \in H$ satisfying $\left(\mathcal{A}\left(x^{(1)}-x^{(2)}\right), x^{(1)}-x^{(2)}\right)<\delta$ and $x^{(1)}-x^{(2)} \in H$ one has $\left|k\left(x^{(1)}\right)-k\left(x^{(2)}\right)\right|<\varepsilon$.

We introduce a dot product in $H:(x, y)_{\mathcal{A}}=(\mathcal{A} x, y)_{H}$. Let $H_{\mathcal{A}}$ be a completion of $H$ with respect to $(\cdot, \cdot)_{\mathcal{A}}$. Let us describe $H_{\mathcal{A}}$ by coordinates. For this purpose we choose an orthonormal basis in $H$ consisting of the eigen vectors of the operator $\mathcal{A}$ realizing $H$ as a space $l_{2}=l_{2}\left(\mathbb{R}^{1}\right)$ with respect to a decomposition in this basic. Then space $H_{\mathcal{A}}$ is realized as a Hilbert space $l_{2}(\mathcal{A})=\left\{x \in \mathbb{R}^{\infty}=\mathbb{R}^{1} \times \mathbb{R}^{1} \times \ldots \mid \sum_{n=1}^{\infty} a_{n} x_{n}^{2}<\infty\right\}$ with the scalar product $(x, y)_{\mathcal{A}}=\sum_{n=1}^{\infty} a_{n} x_{n} y_{n} \in l_{2}(\mathcal{A})\left(\left(a_{n} \delta_{n m}\right)_{n, m=1}^{\infty}\right.$ is a matrix of the operator $\mathcal{A}$ in the considering basis; $a_{n}>0$ ). Let us consider now the standard Gaussian measure $\gamma_{1}=\sqrt{\frac{1}{\pi}} e^{-x^{2}}$, it is essential that $\gamma_{1}\left(l_{2}(\mathcal{A})\right)=1$, (see [8]).

Since $k(x)(x \in H)$ is uniformly continuous with respect to the norm $\|\cdot\|_{H_{\mathcal{A}}}=(\cdot, \cdot)_{H}^{\frac{1}{2}}$ it can be extended by continuity to a uniformly continuous function $k_{1}(x)$ on the whole space $H_{\mathcal{A}}$. Passing to the coordinate from of $H_{\mathcal{A}}$, we find that $k_{1}(x)$ is defined on a set $l_{2}(\mathcal{A}) \subset \mathbb{R}^{\infty}$ of a full Gaussian measure.

Using $k_{1}(x)$ we introduce a quasi-scalar product

$$
\langle\varphi, \psi\rangle=\int_{l_{2}(\mathcal{A})} \int_{l_{2}(\mathcal{A})} \frac{1}{2}\left[k_{1}(x+y)+k_{1}(x-y)\right] \varphi(y) \overline{\psi(x)} d \gamma_{1}(x) d \gamma_{1}(y), \quad\left(\varphi, \psi \in C_{b, c y l}\left(\mathbb{R}^{\infty}\right)\right) .
$$

Then, $H_{\kappa}$ be a Hilbert space obtained as a result of a completion $(\cdot, \cdot)_{H_{\kappa}}=\langle\cdot, \cdot\rangle$. Since the kernel $\frac{1}{2}\left[k_{1}(x+y)+k_{1}(x-y)\right]$ is even on $x, y$, we consider (6) on even functions. Now we will construct a family of unitary operators, acting in $H_{\kappa}$. For this goal we will construct a rigging (chain)

$$
H_{0} \supset H_{+} \supset \mathcal{D} \text {. }
$$

The role of the space $H_{0}$ will be played just by $H_{\kappa}$, namely $H_{0}=H_{\kappa}$ and $H_{+}=L_{2}\left(l_{2}(\mathcal{A}), \mathfrak{B}\left(l_{2}(\mathcal{A}) ; \gamma_{1}\right)\right)$ and $\mathcal{D}$, which consists of the restrictions of functions from the space $\mathcal{A}\left(\mathbb{R}^{\infty}\right)=\bigcup_{n=1}^{\infty} \mathcal{A}\left(\mathbb{R}^{n}\right)$, where $\mathcal{A}\left(\mathbb{R}^{n}\right)=\mathcal{A}\left(\mathbb{R}^{1}\right) \otimes \ldots \otimes \mathcal{A}\left(\mathbb{R}^{1}\right)$ ( $n$ times $)$ and $\mathcal{A}\left(\mathbb{R}^{1}\right)=l_{2}\left(\mathbb{R}^{1}, \gamma_{1}\right)$ on the cylindrical functions. For details of the space $\mathcal{A}\left(\mathbb{R}^{\infty}\right)$ see $[3$, p. 110-113]. The properties of the space $\mathcal{A}\left(\mathbb{R}^{\infty}\right)$ imply the properties of the space $\mathcal{D}$ and the embedding $\mathcal{D} \subset L_{2}=H_{+}$. This embedding is quasi-nuclear. Indeed, the scalar product (6) has the form $(\varphi, \psi)_{H_{\kappa}}=(K \varphi, \psi)_{L_{2}}$, where $K$ is an integral operator in $L_{2}$ generated by the continuous kernel $K(x, y)=\frac{1}{2}[k(x+y)+k(x-y)]$. Since

$$
\int_{l_{2}(\mathcal{A})} K(x, x) d \gamma_{1}(x) \leq k(0)<\infty
$$


this operator is nuclear. On the other hand, for the chain $H_{-} \supset H_{0} \supset H_{+}$, we have $(\varphi, \psi)_{H_{0}}=(\mathrm{I} \varphi, \psi)_{H_{+}}\left(\varphi, \psi \in H_{+}\right)$; therefore $K=O\left(\mathrm{I} \uparrow H_{+}\right)$, and the nuclearity of $K$ yields the quasinuclearity of the embedding $H_{+} \rightarrow H_{0}$. Hence, we have constructed the chain

$$
H_{\kappa}=H_{0} \supset H_{+}=L_{2} \supset \mathcal{D}=\mathcal{A}\left(\mathbb{R}^{\infty}\right) \text {. }
$$

Let us construct a family of unitary operators $\mathcal{A}_{x}\left(x \in L_{2}=H\right)$ acting in the space $H_{\kappa}$. They are the shift operators of functions by $t$ with a certain factor. The factor appears because the Gaussian measure $\gamma_{1}$ is not invariant under such shifts. Let us first define the operator $\mathcal{A}_{t}$ on the function $\varphi \in C_{b, c y l}^{\infty}\left(\mathbb{R}^{\infty}\right)=\bigcup_{n=1}^{\infty} C_{b}^{\infty}\left(\mathbb{R}^{n}\right)$ by the equality

$$
\left(\mathcal{A}_{x} t\right)(x)=\frac{1}{2}\left[\varphi(x-t)\left(\frac{d \gamma_{1,-t}}{d \gamma_{1}}\right)(x)+\varphi(x+t)\left(\frac{d \gamma_{1, t}}{d \gamma_{1}}\right)(x)\right],\left(t \in L_{2} ; x \in l_{2}(\mathcal{A})\right)
$$

where derivative is

$$
\left(\frac{d \gamma_{1, t}}{d \gamma_{1}}\right)(\cdot)=\rho_{\gamma_{1}}(t, \cdot)=\exp \left(-\|t\|_{L_{2}}^{2}-2(t, \cdot)_{L_{2}}\right)
$$

Since for an even kernel $K(x, y)$ and even $\varphi, \psi$ expression (6) has the form

$$
\begin{gathered}
\int_{l_{2}(\mathcal{A})} \int_{l_{2}(\mathcal{A})} \frac{1}{2}[k(x+y)+k(x-y)] \varphi(y) \overline{\psi(x)} d \gamma_{1}(x) d \gamma_{1}(y)= \\
=\int_{l_{2}(\mathcal{A})} \int_{l_{2}(\mathcal{A})} k(y-x) \varphi(y) \overline{\psi(x)} d \gamma_{1}(x) d \gamma_{1}(y)=\int_{l_{2}(\mathcal{A})} \int_{l_{2}(\mathcal{A})} k(x+y) \varphi(y) \overline{\psi(x)} d \gamma_{1}(x) d \gamma_{1}(y),
\end{gathered}
$$

by a simple change of variables we get for any $t \in L_{2}$

Indeed,

$$
\left(\mathcal{A}_{t} \varphi, \mathcal{A}_{t} \psi\right)_{H_{\kappa}}=(\varphi, \psi)_{H_{\kappa}} \quad\left(\varphi, \psi \in C_{b, c y b}^{\infty}\left(\mathbb{R}^{\infty}\right)\right) .
$$

$$
\begin{gathered}
\frac{1}{4} \int_{l_{2}(\mathcal{A})} \int_{l_{2}(\mathcal{A})} k(y-x) \varphi(x-t) \frac{d \gamma_{1,-t}}{d \gamma_{1}}(x) \overline{\psi(y-t)} \frac{d \gamma_{1, t}}{d \gamma_{1}}(y) d \gamma_{1}(x) d \gamma_{1}(y)=\left[\begin{array}{c}
x=x^{\prime}+t ; \\
y=y^{\prime}+t
\end{array}\right]= \\
=\frac{1}{4} \int_{l_{2}(\mathcal{A})} \int_{l_{2}(\mathcal{A})} k\left(y^{\prime}-x^{\prime}\right) \varphi\left(x^{\prime}\right) \frac{d \gamma_{1}}{d \gamma_{1, t}}\left(x^{\prime}\right) \overline{\psi\left(y^{\prime}\right)} \frac{d \gamma_{1}}{d \gamma_{1, t}}\left(y^{\prime}\right) d \gamma_{1, t}\left(x^{\prime}\right) d \gamma_{1, t}\left(y^{\prime}\right)= \\
=\frac{1}{4} \int_{l_{2}(\mathcal{A})} \int_{l_{2}(\mathcal{A})} k\left(y^{\prime}-x^{\prime}\right) \varphi\left(x^{\prime}\right) \overline{\psi\left(y^{\prime}\right)} d \gamma_{1}\left(x^{\prime}\right) d \gamma_{1}\left(y^{\prime}\right) \\
\frac{1}{4} \int_{l_{2}(\mathcal{A})} \int_{l_{2}(\mathcal{A})} k(y-x)\left[\varphi(x-t) \frac{d \gamma_{1, t}}{d \gamma_{1}}(x) \overline{\psi(y+t)} \frac{d \gamma_{1, t}}{d \gamma_{1}}(y)+\right. \\
\left.\left.+\frac{1}{4} \int_{l_{2}(\mathcal{A})} \int_{l_{2}(\mathcal{A})} k(x+t) \frac{d \gamma_{1, t}}{d \gamma_{1}}(x) \overline{\psi(y-t)} \frac{d \gamma_{1,-t}}{d \gamma_{1}}(y)\right] y\right) \varphi(x-t) \frac{d \gamma_{1,-t}}{d \gamma_{1}}(x) \overline{\psi(y+t)} \frac{d \gamma_{1, t}}{d \gamma_{1}}(y) d \gamma_{1}(y)= \\
+\frac{1}{4} \int_{l_{2}(\mathcal{A})} \int_{l_{2}(\mathcal{A})} k(x+y) \varphi(x+t) \frac{d \gamma_{1, t}}{d \gamma_{1}}(x) \overline{\psi(y-t)} \frac{d \gamma_{1,-t}}{d \gamma_{1}}(y) d \gamma_{1}(y)+
\end{gathered}
$$


But

$$
\begin{aligned}
& \frac{1}{4} \int_{l_{2}(\mathcal{A})} \int_{l_{2}(\mathcal{A})} k(x+y) \varphi(x-t) \frac{d \gamma_{1,-t}}{d \gamma_{1}}(x) \overline{\psi(y+t)} \frac{d \gamma_{1, t}}{d \gamma_{1}}(y) d \gamma_{1}(x) d \gamma_{1}(y)=\left[\begin{array}{c}
x=x^{\prime}-t ; \\
y=y^{\prime}+t
\end{array}\right]= \\
& =\frac{1}{4} \int_{l_{2}(\mathcal{A})} \int_{l_{2}(\mathcal{A})} k\left(x^{\prime}+y^{\prime}\right) \varphi\left(x^{\prime}\right) \frac{d \gamma_{1}}{d \gamma_{1,-t}}\left(x^{\prime}\right) \overline{\psi\left(y^{\prime}\right)} \frac{d \gamma_{1}}{d \gamma_{1, t}}\left(y^{\prime}\right) d \gamma_{1,-t}\left(x^{\prime}\right) d \gamma_{1, t}\left(y^{\prime}\right)= \\
& =\frac{1}{4} \int_{l_{2}(\mathcal{A})} \int_{l_{2}(\mathcal{A})} k\left(x^{\prime}+y^{\prime}\right) \varphi\left(x^{\prime}\right) \overline{\psi\left(y^{\prime}\right)} d \gamma_{1}\left(x^{\prime}\right) d \gamma_{1}\left(y^{\prime}\right) \\
& \frac{1}{4} \int_{l_{2}(\mathcal{A})} \int_{l_{2}(\mathcal{A})} k(x+y) \varphi(x-t) \frac{d \gamma_{1,-t}}{d \gamma_{1}}(x) \overline{\psi(y+t)} \frac{d \gamma_{1, t}}{d \gamma_{1}}(y) d \gamma_{1}(x) d \gamma_{1}(y)=\left[\begin{array}{c}
x=x^{\prime}+t ; \\
y=y^{\prime}-t
\end{array}\right]= \\
& =\frac{1}{4} \int_{l_{2}(\mathcal{A})} \int_{l_{2}(\mathcal{A})} k\left(x^{\prime}+y^{\prime}\right) \varphi\left(x^{\prime}\right) \frac{d \gamma_{1}}{d \gamma_{1, t}}\left(x^{\prime}\right) \overline{\psi\left(y^{\prime}\right)} \frac{d \gamma_{1}}{d \gamma_{1,-t}}\left(y^{\prime}\right) d \gamma_{1, t}\left(x^{\prime}\right) d \gamma_{1,-t}\left(y^{\prime}\right)= \\
& =\frac{1}{4} \int_{l_{2}(\mathcal{A})} \int_{l_{2}(\mathcal{A})} k\left(x^{\prime}+y^{\prime}\right) \varphi\left(x^{\prime}\right) \overline{\psi\left(y^{\prime}\right)} d \gamma_{1}\left(x^{\prime}\right) d \gamma_{1}\left(y^{\prime}\right) \\
& \frac{1}{4} \int_{l_{2}(\mathcal{A})} \int_{l_{2}(\mathcal{A})} k(y-x) \varphi(x+t) \frac{d \gamma_{1, t}}{d \gamma_{1}}(x) \overline{\psi(y+t)} \frac{d \gamma_{1, t}}{d \gamma_{1}}(y) d \gamma_{1}(x) d \gamma_{1}(y)=\left[\begin{array}{c}
x=x^{\prime}-t ; \\
y=y^{\prime}-t
\end{array}\right]= \\
& =\frac{1}{4} \int_{l_{2}(\mathcal{A})} \int_{l_{2}(\mathcal{A})} k\left(y^{\prime}-x^{\prime}\right) \varphi\left(x^{\prime}\right) \frac{d \gamma_{1}}{d \gamma_{1,-t}}\left(x^{\prime}\right) \overline{\psi\left(y^{\prime}\right)} \frac{d \gamma_{1}}{d \gamma_{1,-t}}\left(y^{\prime}\right) d \gamma_{1,-t}\left(x^{\prime}\right) d \gamma_{1,-t}\left(y^{\prime}\right)= \\
& =\frac{1}{4} \int_{l_{2}(\mathcal{A})} \int_{l_{2}(\mathcal{A})} k\left(y^{\prime}-x^{\prime}\right) \varphi\left(x^{\prime}\right) \overline{\psi\left(y^{\prime}\right)} d \gamma_{1}\left(x^{\prime}\right) d \gamma_{1}\left(y^{\prime}\right)
\end{aligned}
$$

Summing up (9)-(12), we obtain that $\left(\mathcal{A}_{t} \varphi, \mathcal{A}_{t} \psi\right)_{H_{\kappa}}=(\varphi, \psi)_{H_{\kappa}}\left(\varphi, \psi \in C_{b, c y l}\left(\mathbb{R}^{\infty}\right)\right)$. Thus, each operator $\mathcal{A}_{t}$ is isometric and therefore it may be extended to an isometric operator on the whole of $H_{\kappa}$ by continuity; we preserve the notation $\mathcal{A}_{t}$ for the latter.

Now, we can verify the equality

$$
\left(\mathcal{A}_{t} \varphi, \psi\right)_{H_{\kappa}}=\left(\varphi, \mathcal{A}_{t} \psi\right)_{H_{\kappa}},\left(\varphi, \psi \in C_{b, c y l}\left(\mathbb{R}^{\infty}\right)\right)
$$

Indeed,

$$
\begin{aligned}
& \left(\mathcal{A}_{t} \varphi, \psi\right)_{H_{\kappa}}=\frac{1}{2} \int_{l_{2}(\mathcal{A})} \int_{l_{2}(\mathcal{A})} k(y-x) \varphi(x-t)\left(\frac{d \gamma_{1,-t}}{d \gamma_{1}}\right)(x) \overline{\psi(y)} d \gamma_{1}(x) d \gamma_{1}(y)+ \\
& +\frac{1}{2} \int_{l_{2}(\mathcal{A})} \int_{l_{2}(\mathcal{A})} k(y-x) \varphi(x+t)\left(\frac{d \gamma_{1, t}}{d \gamma_{1}}\right)(x) \overline{\psi(y)} d \gamma_{1}(x) d \gamma_{1}(y)=\left[\begin{array}{c}
x=x^{\prime}+t ; \\
y=y^{\prime}+t
\end{array}\right]= \\
& =\frac{1}{2} \int_{l_{2}(\mathcal{A})} \int_{l_{2}(\mathcal{A})} k\left(y^{\prime}-x^{\prime}\right) \varphi\left(x^{\prime}\right) \frac{d \gamma_{1}}{d \gamma_{1, t}}\left(x^{\prime}\right) \overline{\psi\left(y^{\prime}+t\right)} d \gamma_{1, t}\left(x^{\prime}\right) d \gamma_{1, t}\left(y^{\prime}\right)+\left[\begin{array}{c}
x=x^{\prime}-t ; \\
y=y^{\prime}-t
\end{array}\right]+ \\
& +\frac{1}{2} \int_{l_{2}(\mathcal{A})} \int_{l_{2}(\mathcal{A})} k\left(y^{\prime}-x^{\prime}\right) \varphi\left(x^{\prime}\right) \frac{d \gamma_{1}}{d \gamma_{1,-t}}\left(x^{\prime}\right) \overline{\psi\left(y^{\prime}-t\right)} d \gamma_{1,-t}\left(x^{\prime}\right) d \gamma_{1,-t}\left(y^{\prime}\right)=
\end{aligned}
$$




$$
\begin{gathered}
=\frac{1}{2} \int_{l_{2}(\mathcal{A})} \int_{l_{2}(\mathcal{A})} k\left(y^{\prime}-x^{\prime}\right) \varphi\left(x^{\prime}\right) \overline{\psi\left(y^{\prime}+t\right)} \frac{d \gamma_{1, t}}{d \gamma_{1}}\left(y^{\prime}\right) d \gamma_{1}\left(x^{\prime}\right) d \gamma_{1}\left(y^{\prime}\right)+ \\
+\frac{1}{2} \int_{l_{2}(\mathcal{A})} \int_{l_{2}(\mathcal{A})} k\left(y^{\prime}-x^{\prime}\right) \varphi\left(x^{\prime}\right) \overline{\psi\left(y^{\prime}-t\right)} \frac{d \gamma_{1,-t}}{d \gamma_{1}}\left(y^{\prime}\right) d \gamma_{1}\left(x^{\prime}\right) d \gamma_{1}\left(y^{\prime}\right)=\left(\varphi, \mathcal{A}_{-t} \psi\right)_{H_{\kappa}} .
\end{gathered}
$$

Similarly, we can verify the equality

$$
\left(\mathcal{A}_{t} \varphi, \mathcal{A}_{s} \psi\right)_{H_{\kappa}}=\frac{1}{2}\left[\left(\mathcal{A}_{t-s} \varphi, \psi\right)_{H_{\kappa}}+\left(\mathcal{A}_{t+s} \varphi, \psi\right)_{H_{\kappa}}\right]
$$

It implies that $\mathcal{A}_{t}=\mathcal{A}_{t}^{*}=\mathcal{A}_{-t}$, and $\mathcal{A}_{t} \cdot \mathcal{A}_{s}=\frac{1}{2}\left[\mathcal{A}_{t+s}+\mathcal{A}_{t-s}\right]\left(t, s \in l_{2}\right)$. Hence, we have constructed the group of unitary operators $\left(\mathcal{A}_{t}\right)_{t \in l_{2}}$. These operators and the chain (6) are connected by the conditions 1,2 of Theorem $1.2[4$, Ch. 4]. Therefore, we can write the representation

$$
\mathcal{A}_{x}=\int_{X} e^{i(\lambda, x)} d E(\lambda), \quad(x \in X),
$$

for the introduced operators $\mathcal{A}_{x}$ in the space $H_{\kappa}$. Here, $E$ is a decomposition of unity on $\mathfrak{B}(X)$.

But so as $\mathcal{A}_{x}=\mathcal{A}_{-x}$, we can write such representation

$$
\mathcal{A}_{x}=\int_{H} \cos (\lambda, x) d E(\lambda), \quad(x \in H) .
$$

For any $x \in X_{\mathcal{A}}$, the space $H_{\kappa}$ includes $\delta$-function $\delta_{x}$, concentrated at the point $x$, and $\left(\delta_{x}, \delta_{y}\right)_{H_{\kappa}}=\frac{1}{2}\left[k_{1}(x-y)+k_{1}(x+y)\right]\left(x, y \in X_{\mathcal{A}}\right)$. More precisely, for a given negative space $H_{\kappa}$ and zero space $L_{2}$, we can construct a chain $H_{\kappa} \supset L_{2} \supset H_{+}$. As a result, we obtain the space $H_{+}$consisting of continuous bounded functions on $X_{\mathcal{A}}$. The space $H_{\kappa}$ includes vectors $\delta_{x}$ such that $\left(\delta_{x}, \varphi\right)_{L_{2}}=\overline{\varphi(x)}\left(x \in X_{\mathcal{A}}, \varphi \in H_{+}\right)$and the indicated equality holds.

It is easy to understand that $\mathcal{A}_{t} \delta_{0}=\delta_{t}$ for any $t \in X$. Indeed, let $\left(\chi_{n}(\cdot)\right)_{n=1}^{\infty}$ be a sequence of functions from $L_{2}$ which converges to $\delta_{0}$ in $H_{\kappa}$, and let each of these functions be equal to the characteristic function of the ball centered at $\mathrm{O}$ in $X_{\mathcal{A}}$ with radius $n^{-1}$ divided by the measure $\gamma_{1}$ of this ball. Clearly, the action of the operator $\mathcal{A}_{t}$ on $\chi_{n}$ is given by (7).

For $\varphi \in H_{+}$we have

$$
\begin{gathered}
\left(\mathcal{A}_{t} \delta_{0}, \varphi\right)_{L_{2}}=\lim _{n \rightarrow \infty}\left(\mathcal{A}_{t} \chi_{n}, \varphi\right)_{L_{2}}=\lim _{n \rightarrow \infty}\left[\int_{H_{\mathcal{A}}} \frac{1}{2} \chi_{n}(x-t) \frac{d \gamma_{1,-t}}{d \gamma_{1}}(x) \overline{\varphi(x)} d \gamma_{1}(x)+\right. \\
\left.+\int_{H_{\mathcal{A}}} \frac{1}{2} \chi_{n}(x+t) \frac{d \gamma_{1, t}}{d \gamma_{1}}(x) \overline{\varphi(x)} d \gamma_{1}(x)\right]= \\
=\lim _{n \rightarrow \infty}\left[\int_{H_{\mathcal{A}}} \frac{1}{2} \chi_{n}(x-t) \overline{\varphi(x)} d \gamma_{1,-t}(x)+\int_{H_{\mathcal{A}}} \frac{1}{2} \chi_{n}(x+t) \overline{\varphi(x)} d \gamma_{1, t}(x)\right]= \\
=\lim _{n \rightarrow \infty}\left[\int_{H_{\mathcal{A}}} \frac{1}{2} \chi_{n}(x) \overline{\varphi(x+t)} d \gamma_{1}(x)+\int_{H_{\mathcal{A}}} \frac{1}{2} \chi_{n}(x) \overline{\varphi(x-t)} d \gamma_{1}(x)\right]=\overline{\varphi(t)}=\left(\delta_{t}, \varphi\right)_{L_{2}},
\end{gathered}
$$


and this yields the equality $\mathcal{A}_{t} \delta_{0}=\delta_{t}$.

After these remarks, representation (3) immediately follows from (15). One should apply the last equality to $\delta_{0}$ and multiply by a scalar $\delta_{0}$ in $H_{\kappa}$. Here $\sigma(\alpha)=\left(E(\alpha) \delta_{0}, \delta_{0}\right)_{H_{\kappa}}$ $(\alpha \in \mathfrak{B}(X))$. Theorem 1 is proved.

Applying Theorem 1 we can prove the following theorem.

Theorem 2. In order that a family $\left(\mathcal{A}_{t}\right)(t \in H)$ of self-adjoint bounded operators in the Hilbert space $H$ admits a representation

$$
\mathcal{A}_{t}=\int_{H} \cos (\lambda, t) d E(\lambda), \quad(\lambda \in H),
$$

where $E$ is some decomposition of identity of $\mathfrak{B}(H)$, it is necessary and sufficient that the operators satisfy the following conditions:

1) $\frac{1}{2}\left[\mathcal{A}_{t+s}+\mathcal{A}_{t-s}\right]=\mathcal{A}_{t} \mathcal{A}_{s}, \quad \mathcal{A}_{\left(t_{1}, \ldots, t_{n}, \ldots\right)}=\mathcal{A}_{\left(t_{1}, \ldots,-t_{n}, \ldots\right)} ; \quad \mathcal{A}_{0}=\mathrm{I} ;$

2) $\mathcal{A}_{t}$ strongly continuously depends on $t$;

3) $\left\|\mathcal{A}_{t}\right\| \leq b<\infty$.

Proof. Sufficiency. Let operators $\mathcal{A}_{t}$ commute accordingly to 1) and have a common simple spectrum. Then there exists a cycle vector $\Omega$ such that a closed linear hull $\left\{\mathcal{A}_{t}, \Omega\right\}=H$. Put $\left(\mathcal{A}_{t} \Omega, \Omega\right)=k(t)$. Then $k(t)$ is continuous and even in every variable and e.p.d., bounded function on $H$. Indeed, the function $k(t)$ is e.p.d.

$$
\begin{gathered}
\sum_{i, j=1}^{n} \frac{1}{2}\left[k\left(x^{(i)}+x^{(j)}\right)+k\left(x^{(i)}-x^{(j)}\right)\right] \xi_{i} \overline{\xi_{j}}=\sum_{i, j=1}^{n} \frac{1}{2}\left[\left(\mathcal{A}_{t^{(i)}+t^{(j)}} \Omega, \Omega\right)+\left(\mathcal{A}_{t^{(i)}-t^{(j)}} \Omega, \Omega\right)\right] \xi_{i} \overline{\xi_{j}}= \\
=\sum_{i, j=1}^{n} \frac{1}{2}\left[\left(\mathcal{A}_{t^{(i)}} \mathcal{A}_{t^{(j)}} \Omega, \Omega\right)+\left(\mathcal{A}_{t^{(j)}} \Omega, \mathcal{A}_{t^{(i)}} \Omega\right)\right] \xi_{i} \overline{\xi_{j}}=\left\|\sum_{i=1}^{n} \xi_{i} \mathcal{A}_{t^{(i)}} \Omega\right\|_{H}^{2} \geq 0 .
\end{gathered}
$$

The continuity, evenness in every variable and boundedness of the function $k(t)$ follow from assumptions of Theorem 2. Therefore by Theorem 1, we obtain an integral representation for the function $k(t)$

$$
k(t)=\int_{H} \cos (\lambda, t) d \rho(\lambda), \quad(t \in H),
$$

where $d \rho(\lambda)$ is an even non-negative finite measure, which is defined on some $\sigma$-algebra $\mathfrak{B}(H)$ of Borel sets from $H$. We construct a correspondence: every vector $\sum_{\kappa=1}^{n} c_{\kappa} \mathcal{A}_{t^{(\kappa)}} \Omega \in H$ is paired with the function

$$
\sum_{\kappa=1}^{n} c_{\kappa} \cos \left(\lambda, t^{(\kappa)}\right) \in l_{2}(H, d \rho(\lambda)), \quad(n=1,2, \ldots) .
$$

This correspondence is isometric because

$$
\left\|\sum_{\kappa=1}^{n} c_{\kappa} \mathcal{A}_{t_{\kappa}} \Omega\right\|_{H}^{2}=\sum_{i, j=1}^{n} c_{i} \overline{c_{j}}\left(\mathcal{A}_{t_{i}} \Omega, \mathcal{A}_{t_{j}} \Omega\right)_{H}=\sum_{i, j=1}^{n} c_{i} \overline{c_{j}} \frac{1}{2}\left[\left(\mathcal{A}_{t_{i}+t_{j}} \Omega, \Omega\right)_{H}+\left(\mathcal{A}_{t_{i}-t_{j}} \Omega, \Omega\right)_{H}\right]=
$$




$$
\begin{gathered}
=\sum_{i, j=1}^{n} c_{i} \overline{c_{j}} \int_{H} \frac{1}{2}\left[\cos \left(\lambda, t^{(i)}+t^{(j)}\right)+\cos \left(\lambda, t^{(i)}-t^{(j)}\right)\right] d \rho(\lambda)= \\
=\left\|\sum_{\kappa=1}^{n} c_{\kappa} \cos \left(\lambda, t^{(\kappa)}\right)\right\|_{l_{2}(H, d \rho(\lambda))}^{2} \cdot
\end{gathered}
$$

Then it can be extended to the unitary map $U: H \rightarrow l_{2}(H, d \rho(\lambda))$. Furthermore, the operator $\mathcal{A}_{t}$ corresponds to the operator of multiplication in $l_{2}(H, d \rho(\lambda))$ on the function $\cos (\lambda, t)$.

Since

$$
\begin{gathered}
\mathcal{A}_{t}\left(\sum_{\kappa=1}^{n} c_{\kappa} \mathcal{A}_{t^{(\kappa)}} \Omega\right)=\sum_{\kappa=1}^{n} c_{\kappa}\left(\frac{\mathcal{A}_{t+t^{(\kappa)}}+\mathcal{A}_{t-t^{(\kappa)}}}{2}\right) \Omega= \\
=U^{*}\left(\sum_{\kappa=1}^{n} c_{\kappa} \frac{\cos \left(\lambda, t+t^{(\kappa)}\right)+\cos \left(\lambda, t-t^{(\kappa)}\right)}{2}\right)= \\
=U^{*} \cos (\lambda, t) U\left(\sum_{\kappa=1}^{n} c_{\kappa} \cos \left(\lambda, t^{(\kappa)}\right)\right)=U^{*} \cos (\lambda, t) U\left(\sum_{\kappa=1}^{n} c_{\kappa} \mathcal{A}_{t^{(\kappa)}} \Omega\right),
\end{gathered}
$$

that is $\mathcal{A}_{t}=U^{*} Q_{\cos (\lambda, t)} U\left(Q_{\cos (\lambda, t)}\right.$ denotes the operator of multiplication on $\cos (\lambda, t)$ in $\left.l_{2}(H, d \rho(\lambda))\right)$.

Since for a family $Q_{\cos (\lambda, t)}$ the integral representation is true

$$
Q_{\cos (\lambda, t)}=\int_{H} \cos (\lambda, t) d Q_{\chi_{\Delta}(\cdot)}
$$

we have $\mathcal{A}_{t}=\int_{H} \cos (\lambda, t) d E(\lambda)$, where $E(\Delta)=U^{*} Q_{\chi_{\Delta}(\cdot)} U . \quad \chi_{\Delta}(\cdot)$ is the characteristic function of an interval $\Delta \in H$. So the integral representation (16) is proved.

The uniqueness of decomposition of identity follows from the uniqueness of the measure $d \rho(\lambda)$. If the cyclic vector $\Omega$ is missed then for the family of commuted self-adjoint operators $\mathcal{A}_{t}(t \in H)$ in $H$ there exists a sequence of measures on $\sigma$-algebra of cylindric sets in $H$ with a Borel basis $\rho_{1}, \ldots, \rho_{\kappa}, \ldots$ and a unitary operator

$$
U: H \rightarrow \oplus \sum_{\kappa=1}^{\infty} l_{2}\left(H, d \rho_{\kappa}(\lambda)\right)
$$

such that in every space $l_{2}\left(H, d \rho_{\kappa}(\lambda)\right)$ one has $\mathcal{A}_{t}=U^{*} Q_{\cos (\lambda, t)} U$, where $Q_{\cos (\lambda, t)}$ denotes the operator of multiplication on $\cos (\lambda, t)$ in $l_{2}\left(H, d \rho_{\kappa}(\lambda)\right)$.

The necessity of the conditions 1), 2), 3) follows from integral representation (16).

An integral representation for the family of bounded self-adjoint operators in one-dimensional case, which are connected by an algebraic relationship, was proved in [6]. 


\section{REFERENCES}

1. Berezansky Yu.M. Generalization of Bochner theorem on expansions in eigenfunctions of partial differential operators// Dokl. AN SSSR. - 1956. - V.110, no.6. - P. 893-896.

2. Berezansky Yu.M. Expansions in eigenfunctions of self-adjoint operators. (Translations of Mathematical Monographs V.17), Providence, R.I.: Am. Math. Soc., 1968, 809 p.

3. Berezansky Yu.M. Self-adjoint operators in space of functions of infinitely many varibles. - Kyiv: Naukova dumka, 1978. - $360 \mathrm{p}$.

4. Berezansky Yu.M., Kondratiev Yu.G. Spectral methods in infinite-dimensional analysis. - Kyiv: Naukova dumka, 1988. - 679 p. Engl.transl.: Springer, Dordrecht. 1995, doi: 10.1007/978-94-011-0509-5

5. Krein M.G. On a general method on decomposition of Hermite positive definite nuclei into elementary products// Dokl. AN SSSR. - 1946. - V.53(1). - P. 3-6.

6. Kurepa S.A. A cosine functional equation in Hilbert space // Canadian J. Math. - 1960. - V.12. - P. 45-50.

7. Minlos R.A. Generalized random processes and their extension in measure// Trudy Moskov. Mat. Obsc. - 1959. - V.8. - 497-518. (in Russian)

8. Shilov G.E., Fan Dyk Tin. Integral, measure and derivative on linear spaces. - M.: Science, 1967. - 192 p.

9. Sazonov V.V. Remark on characteristic functionals// Theory of Probability and its Applications. - 1958.

- V.3, no.2. - P. 188-192. doi: 10.1137/1103018

National Forestry and Wood Technology University of Ukraine

Lviv, Ukraine

Lopotko1304@gmail.com

Received 24.04.2020

Revised 18.12.2020 